\title{
LAS IDEAS DE BLANCO WHITE SOBRE SHAKESPEARE *
}

\author{
Miguel Angel Cuevas \\ Universidad de Alicante
}

La etapa final de la vida de Blanco White es, si no llevamos demasiado lejos la comparación, la gran desconocida. Usualmente se ha visto despachada de un plumazo con las consabidas referencias a su abandono del anglicanismo, su refugio afectivo entre los unitarios de Liverpool y el progresivo avance de una enfermedad que culminaría en 1841. Sin embargo, éste es, si cabe, el momento más radicalmente trascendental de la existencia de nuestro autor. Desde el punto de vista literario, se deben a las especulaciones ideológicas de estos años algunos de los

\footnotetext{
* Este artículo es adelanto y sintesis de una de las partes de mi tesis doctoral, en preparación , sobre las ideas literarias de José María Blanco White. En atención a la mejor comprensión del trabajo, se ha intentado evitar la referencia a obras anteriores a las que nos ocupan en la producción de nuestro autor; con todo, habida cuenta de la imposibilidad de ejercer totalmente tal premisa, pido disculpas en este sentido. Los textos de Blanco se dan siempre en su lengua original y, en su caso, se traducen en nota encerrando la versión española entre corchetes; las traducciones a las que no siga indicación alguna de procedencia son versiones del autor de este trabajo.
} 
escritos donde se muestra más a las claras cuál es la vía - si autónoma de afecciones explícitas exclusivas no por ello menos trasparente para un observador mínimamente familiarizado con Blanco- que se ha desarrollado progresiva y orgánicamente desde muy temprano. En cuanto al aspecto religioso, cuya conspicua recurrencia exige proporcional atención, es de esta época el máximo legado que Blanco ha propuesto; desde las Observations on Heresy and Orthodoxy (1835) hasta las páginas de reflexión religiosa insertas en los últimos Private Journals (1) - con todo lo que tales escritos manifiestan de independencia de criterios, de renuncias a bien aseguradas bases de continuidad en el credo oficial, de búsqueda ininterrumpida de una religiosidad fundamentada racionalmente-, nuestro autor concluye en la más sintomáticamente confusa de las aserciones religiosas del hombre moderno: quedándose solo frente a cualquier confesión, frente a toda «iglesia» (incluso ante los unitarios), en un desnudo y agónico deísmo. (2)

Toda referencia a los trabajos literarios de esta etapa definitiva ha de comenzar por una bipartición. Un primer grupo de textos aparece entre los años 1835 y 1836 en The London Review, que dirigia John Stuart Mill (3): de ellos son interesantes para nuestro propósito uno sobre Martínez de la Rosa y otro sobre el teatro isabelino británico (4). Por otra parte, a partir de 1839 se produce la postrera exposición de las ideas estéticas de Blanco en varios trabajos, entre los que destacan los cuatro dedicados exclusivamente a Shakespeare, publicados en Christian Teacher, revista de la comunidad unitaria de Liverpool. (5)

(1) Insertos en The Life of the Rev. Joseph Blanco White, written by himself, with portions of his correspondence. Edited by John Hamilthon Thom. London, 1845, 3 vols. (Version española del primer volumen "Narrative of his Life in Spain and England". en Autobiografla de Blanco White, edición, traducción, prólogo y notas de Antonio Garnica, Universidad de Sevilla, 1975).

(2) Un cumplido análisis de la evołución religiosa de Blanco White a través de sus obras impresas y de los escritos privados (algunos de los cuales permanecen inéditos en inglés) no es motivo del presente trabajo. Las anotaciones que se hacen en este sentido, aparte de ser puramente referenciales, poseen un inherente carácter de provisionalidad dada su no pretendida naturaleza exhaustiva. Esta parcela de la obra de Blanco espera y exige un detenido estudio autónomo, ante cuyas virtuales evidencias podrian adquirir la certeza necesaria los datos vertidos ahora.

(3) The London Review es la misma revista que, a partir de su tomo tercero (1836), se conoce como London and Westminster Review.

(4) «Recent Spanish Literature. Obras literarias de Don Francisco Martinez de la Rosa», The London Review, I, $n^{\circ} 1$ (1835), pp. 76-93. "Lamb's Specimens of English Dramatic Poets", Ibid, II, $n^{\circ} 3$ (1835-1836), pp 51-69. (Traducciones parciales en José María Blanco White, Obra inglesa, selección y traducción de Juan Goytisolo, Buenos Aires, 1972, pp. $310-319$ y $320-323$ respectivamente) 
Ciertamente se nota en los escritos de Blanco una seguridad expositiva, producto de una madurada reflexión, que excede a un cierto tono de timidez observable en obras anteriores. Al margen de que su propia evolución lo ha llevado a posturas en las que se instala ahora más firmemente, hay que pensar también en el reconocimiento que ha supuesto para él ser llamado a colaborar en una de las revistas importantes de la reciente intelectualidad británica, The London Review, capitaneada por el que puede considerarse uno de los principales ensayistas de la segunda fase de exposición de la teoría expresiva anglosajona. En efecto, John Stuart Mill será el encargado de desarrollar las sugerencias del Wordsworth de 1800 en dos ensayos capitales que publica en 1833: ¿Qué es la poesía? y Las dos clases de poesía (6), escritos en los que se sistematizan varios conceptos característicos del momento y se llevan otros hasta formulaciones extremas: la superioridad de la lírica sobre cualquier otro tipo de poesía, la relegación a un plano muy alejado del "asunto» setecentista, la superación de los objetos exteriores para buscar la auténtica poesía en la emoción expresada, la constitución de la obra como símbolo autónomo y, por fin, la absoluta pérdida de relevancia del auditorio como sujeto paciente de la obra literaria. Tal desarrollo se halla mucho más cercano del idealismo de Coleridge que de las apreciaciones del autor de Lyrical Ballads; y en la base de ambos pueden entreverse las teorías alemanas del genio orǵanico y de la cerrada autoordenación del producto literario. Blanco White había instrumentalizado en alguna medida las ideas de Coleridge en escritos anteriores; los postulados de Mill no podían, por tanto, resultarle extraños, y así se mostrará en sus artículos últimos al hacer propio alguno de ellos. De otro lado, los conceptos expuestos por Mill estaban ya en el ambiente y no cabe imputar al joven filósofo excesiva originalidad en la argumentación concreta sino en el desarrollo sistematizado del conjunto.

La conferencias que August Wilhelm Schlegel pronunciara en Viena

(5) "The Pictorial Shakspere», Christian Teacher, I (1839), pp. 322-332; "The Pictorial Shakspere. Second notice", Ibid., pp. 469-481; "The Pictorial Shakspere. Notes on Hamlet", Ibid., pp. 573-580; Christian Teacher, II (1840), Pictorial Shakspere. A Midsummer Night's Dream», pp., $42-53$ (traducción parcial de Goytisolo, op. cit. pp., 319 y 326). Sobre la inusual forma de escribir el nombre del dramaturgo inglés, el mismo Blanco nos dice en nota a pie de página en la primera del artículo inicial de la serie: "We adopt this spelling in consequence of the proofs which the editor [de The Pictoria/ Shakspere] gives, that the great Poet used thus to write his own namen. [Adoptamos esta grafía de acuerdo con las pruebas que el editor ofrece de que el gran poeta solia escribir así su propio nombre].

(6) Incluidos en Essays by John Stuart Mill, London 1897. 
entre 1809 y 1811 (desarrollo de las primeras berlinesas de 1801-1804), Über Dramatische Kunst und Literatur, habían sido traducidas al inglés en 1815 como Lectures on Dramatic Arts and Literature, y Blanco ya las ha nombrado en un artículo de 1824 a propósito de Calderón en el que se muestra disconforme con lo que juzga exagerada admiración de los autores alemanes por el dramaturgo español (7). Ahora, sin embargo, nuestro autor va a tomar contacto directo con la filosofía idealista alemana mediante la traducción que realiza en 1837 de la parte inicial de Grundzuege zur System der Philosophie de Immanuel Hermann Fichte, hijo de Johann Gottlieb. (8) De esta forma, en 1840, en el último artículo que escribió sobre Shakespeare, Blanco tiene a la vista la traducción alemana que A.W. Schlegel hizo del dramaturgo inglés durante la primera década del siglo XIX, anotada y editada en torno a 1820 por Ludwig Tieck.

El conocimiento que tiene Blanco White del idealismo estético alemán es, pues, de primera mano y sus apreciaciones no pueden considerarse como un eco mimético de Coleridge y John Mill, pese a que conociera y se sintiera atraído, por supuesto, por las obras de los autores británicos. Puede incluso pensarse que las teorías estéticas idealistas de tales ensayistas actuaran en alguna medida como revulsivo para que se preocupara por aprender la lengua alemana a partir de 1833. (9) En cualquier caso, la presencia en el momento de temas que ya han sido tratados en textos más antiguos (así el problema de la verosimilitud o el de las relaciones entre naturaleza y arte) está fundamentando la presunción de un desarrollo a partir de actitudes previas no modificado sustancialmente por cuestiones de puesta al día obligadas por el ambiente cultural. Formulaciones como las que ahora se nos presentan ya han sido articuladas - siquiera en germen - en otros trabajos; $y$ no se contradice esencialmente el tratamiento dado en diferentes épocas a temas recurrentes. En ésta final se los lleva hasta sus últimas consecuencias y se acentúa algún aspecto - lo que confiere radicalidad y mayor seguridad teórica a los asertos. Así sucede, por ejemplo, con el concepto de la naturaleza simbólica de la obra literaria, que ahora adquiere una más amplia configuración aplicado a la esencia de la representación teatral

(7) "Garcilaso en ingles", Variedades o mensagero de Londres, I, $n^{\circ} 5$ loctubre de 1824), pp. 435ss.

(8) Esta traducción parcial que Blanco titula Theory of Cognition, se conserva inédita en el Manchester College de la Universidad de Oxford. Cf. Vicente Lloréns, prólogo a José María Blanco White, Antología de obras en español, Barcelona, 1971, p. 60.

(9) Hecho en el que tuvo especial influencia su inquietud religiosa, que le indujo a leer directamente al téologo germano August Naender. 
que, en una paradigmática formulación, se considera privadora de la captación de la idealidad poética por su apariencia estrechamente realista; lo que hace que Blanco White proponga la vuelta del teatro a su genuino carácter de mero espectáculo. De tal índole son las evoluciones teóricas seguidas por el escritor sevillano.

Antes de pasar a comentar los cuatro artículos de Christian Teacher en los que Blanco se encarga de analizar aisladamente algunas producciones dramáticas de Shakespeare, es conveniente conocer sus anotaciones previas sobre el escritor británico a fin de valorar en su justa medida la profundidad y contundencia de las interpretaciones ulteriores. En el primer número de su revista Variedades o mensagero de Londres (10) Blanco había ofrecido a sus lectores unas versiones castellanas de fragmentos del dramaturgo inglés $y$ al introducirlas hablaba de la dificultad de las traducciones; al explicar la razón por la que, pese a todo, él daba las suyas, anotaba:

[...] nuestra intencion es dar alguna idea a los lectores Españoles, no de las bellezas de Shakspeare, sino del tono de sus pensamientos, y la originalidad de su ingenio. Queremos ademas precaverlos contra las declamaciones de los autores Franceses que hablan de Shakspeare, como de un loco o extravagante porque, parandose solo en los pasages que verdaderamente son defectuosos, no se hallan capaces de apreciar los que son inimitables. El gran poeta Ingles no se sujetó a otras reglas que a las impresiones poderosas y vivisimas de su alma. El teatro estaba lleno de delirios, y Shakspeare se dexó llevar de la corriente; pero, hasta sus delirios son pruebas de un genio poderosisimo, y de un talento incomparable. (11)

A la luz de los textos aducidos pudiera aún pensarse que la admiración de nuestro autor por el dramaturgo inglés no iba más allá que la de

(10) Variedades o mensagero de Londres, Londres, 1823-1825. Consta de dos tomos con un total de nueve números, redactados en su casi total integridad por el emigrado español.

(11) "Shakspeare: traduccion poética de algunos pasages de sus dramas», Variedades, $\mathrm{I}, \mathrm{n}^{\circ} 1$ (enero de 1823), pp. 74ss. En su artículo posterior publicado igualmente en Variedades ("Revisión de obras. El testimonio vengado, comedia de Lope de Vega", I, n 3 (abril de 1824), pp. 247-254), anota Blanco: «[...] En este punto, es decir, en descubrir los secretos del corazon, y en presentar a los personages Dramaticos como si llevasen un crystal en el pecho, que dexase ver todos los movimientos y operaciones del alraa; no tiene igual el poeta Ingles Shakspearen (p. 249). 
cualquier preceptista de última hora que, reconociendo la genialidad innata del autor de Hamlet, lamentara la no sujeción de su capacidad creadora a las normas universales del drama; así se explicaría la presencia de pasajes memorables (dicho sea con un punto de resquemor indisimulado por el forzoso reconocimientol al lado de extravagantes monstruos que atentan contra las más permisivas actitudes de la razón humana. Un pensamiento que encaja a la perfección con la furibunda manía de algún que otro falso y segundón reformador de los que se dedicaban a ajustar las antiguas comedias a los patrones nuevamente impuestos, remedando las tareas indumentarias de los pícaros. Un pensamiento que, si no de forma tan grosera, es posible, por ejemplo, en el ecléctico Mendíbil o en el digno Moratín exiliado en París. Pero no es éste, ni lejanamente, el planteamiento de Blanco White; es más bien el suyo el de un culturalista en fase de evolución que va sintiendo progresiva admiración por el genio británico y que, sin necesidad de omitir su impresión - producto presumible, bien que sólo en parte, de la suerte de encrucijada estética en que se encuentra-, reconoce en él algún fallo que no imputa al abandono de las reglas clásicas sino al viciado y ampuloso estilo teatral de la época.

Veamos otra breve referencia que habrá de servir para ratificar lo apuntado. La encontramos en el artículo citado sobre Martínez de la Rosa, donde se clarifica por completo la posición de Blanco White y se despacha de paso cualquier posible errónea interpretación:

[...] He [Martínez de la Rosa] has, indeed, too much taste not to grand a high degree of merit to Shakspeare; but whatever offends him in his plays is attributed to the great poet's ignorance or contempt of Aristotle. (12)

Aún encontramos otra referencia a Shakespeare anterior a los ensayos de 1839-40. En un artículo sobre el teatro isabelino se detiene Blanco a comentar la figura del dramaturgo y a marcar las discrepancias entre su obra y la de sus coetáneos. Se observa todavía en estas notas cierta reticencia - afín a la de la época de Variedades - que ha de ceder, como veremos, en seguida:

(12) The London Review, $1, \mathrm{n}^{\circ} 1$ (1835), p. 81. "Tiene en verdad demasiado buen gusto para no conceder gran importancia a Shakespeare; pero cuanto le molesta en sus comedias lo achaca a su ignorancia o desprecio de Aristóteles". (Traducción en Goytisolo, Obra inglesa, p. 313/]. 
It is undeniable that the old English dramatists have left some beautiful passages, buried in an immense mass of extravagance and absurdity. In Shakspeare's works there is such a profusion of beauty - the bursts of genuis are so frequent - the glow of life which pervades even the most defective parts, is so attractive, that we completely forget the essentially wrong plan upon which he gave himself up to the spontaneous impulses of his wonderful mind. But no poet inferior to him could follow that path with success; no genius below his could employ the external forms of the style prevalent in those days, especially on the theatre, and still retain the esplendour of native beauty. (13)

Luego de estas incompletas, parciales notas, pasamos ya a comentar los escritos que han sido presentados como definitivo motivo de referencia. Vale decir, en primer lugar, que constituyen el cuerpo de crítica aplicada más importante de la obra de Blanco, superando en número e intensidad a cuantos otros temas fueron abordados por su pluma. Sin embargo, el hecho de que dichos artículos fueran publicados en una revista de ámbito muy restringido ha impedido que hasta ahora hayan sido estudiados con el mínimo detenimiento. Alfonso Par los desconocía al ocuparse de las ideas sobre Shakespeare de Blanco (14), dado lo cual hubo de limitar su documentación a los diarios privados que editara póstumamente Hamilton Thom. Con todo, las anotaciones de Par son valorables por cuanto representan el primer contacto de la crítica especializada con el pensamiento literario último del publicista español; su juicio general sobre los comentarios de Blanco es acertado (15); traduce, ade-

(13) The London Review, II, $\mathrm{n}^{\circ} 3$ (1835-36), pp. 55ss. [ «Es indudable que los antiguos dramaturgos ingleses han dejado algunos pasajes hermosos, mezclados en una masa inmensa de extravagancias y absurdos. En las obras de Shakespeare hay tal profusión de belleza, las llamaradas de genialidad son tan frecuentes, el colorido de vida que penetra hasta en las partes más defectuosas es tan atractivo, que olvidamos por completo el plan esencialmente erróneo por el que se guía y la total negligencia y desatención con la que se entrega a los espontáneos impulsos de su maravillosa inteligencia. Pero ningún poeta inferior puede seguir este camino con éxito; ninguna fuerza creadora inferior a la suya podría emplear las formas externas del estilo que prevalecía en su tiempo, especialmente en el teatro, y mantener el esplendor de la belleza origínal»)l.

(14) Alfonso Par, Shakespeare en la literatura española, 2 vols., Madrid-Barcelona, 1935, 1, pp. 195-203.

(15) Cf., Alfonso Par, op. cit., l, p. 199: «En cuanto a su criterio sobre al dramaturgo inglés, observaré que por primera vez encontramos un literario español que lo conoce intimamente y lo saborea con fruición en términos que no se para en alabanzas generales, sino que adelanta juicios concretos y trata de desentrañar aquellas expresiones geniales que tanto hacen sentir y tan difíciles son de analizar». 
más, algunos fragmentos de sus borradores, uno de los cuales es de especial interés para nuestro propósito.

Pero vayamos por partes. Ya se conoce cómo Blanco, a partir de sus notas en Variedades y sobre todo en torno a los años de su colaboración en la revista de John Stuart Mill, ha ido acercándose a Shakspeare degustánidolo cada vez más placenteramente; mas, por lo que sabemos, aún no ha conseguido eliminar algún ligero disgusto - siquiera reflexionando teóricamente a posteriori - frente a la pervertida estética teatral del período isabelino que, en menor medida que a otros escritores, ha afectado algo al dramaturgo inglés. Su punto de vista inicia una modificación en 1837. En unas anotaciones privadas de 16 de marzo, donde además reflexiona sobre su propia formación cultural, leemos:

Para quien el prototipo del gusto fueron siempre los clásicos especialmente si estudió los escritores franceses anteriores a la Revolución, la dificultad principal de Shakespeare no consiste tanto en la falta de las unidades como en la novedad y atrevimiento de sus metáfotas. Es precisa una familiaridad perfecta con el mundo que vive en la imaginación del poeta, para adivinar en seguida las analogías de donde proceden aquéllas. En su carácter y forma externas tales metáforas son tan semejas al lenguaje figurado eufuista, que quien conozca y deteste las composiciones extravagantes de algunos poetas italianos y españoles, sentirá una aversión instintiva hacia muchos pasajes de Shakespeare, meramente a causa de tal parecido externo. Pero la diferencia entre la vacuidad de aquéllos y la riqueza verdadera y natural del poeta inglés es inmensa... El eufuista busca la novedad ciega y extravagantemente; Shakespeare la encuentra sin esfuerzo, por su inspiración genial. Sus metáforas están repletas de la vida más vigorosa; revela los lazos secretos con que la naturaleza relaciona las nociones, en apariencia, más distantes.

Debe reconocerse, empero, que yerra en algunos casos en que se expansiona hacia una hinchazón que en su tiempo había empezado ya a corromper el gusto de Europa... (16)

Tal cambio de perspectiva - por cuanto muestra mayor precisión en la indagación de la idiosincrasia del drama shakespeareano, natural y genial, frente al amanerado y tardo común en la época - viene acompa-

(16) Apud, ibid. pp. 200ss. 
ñado todavia de cierto recelo, que teminará por transformarse en entera admiración, veneración casi, en el primero de los cuatro artículos que constituyen la serie del Pictorial Shakspere; donde además va a haber una llamada explícita, que diriase que Blanco podría en cierta forma dirigir retrospectivamente a si mismo, a quienes han olvidado que es necesaria una intima penetración en las definitivamente geniales creaciones del poeta británico para que sus apariencias no resulten en nada comparables al estilo vulgar y afectado de obras coetáneas:

Shakspere is a writer whose poetical beauties cannot be properly enjoyed except by those who have studied him. Like Michael Angelo - the Shakspere of painters, - and like all truly, inspired artists, our poet displays none of those superficial, and almost meretricious charms, which, at once, captivate the eye of the vulgar. Without assiduous worship in the sanctuary of Genius, let no one expect that he shall be made partaker of ist revelations. He will, on the contrary, be punished for his temerity, by finding those externals, which are manifest to the common eye, not only indifferent, but repulsive. (17)

En cuanto a las ideas generales que informan su crítica, Blanco no sólo es consciente de que se encuentran muy distantes de las nociones del clasicismo francés (18) sino que también explicita su desacuerdo con el gran crítico shakespeareano británico del siglo anterior Samuel Johnson. Una de las veces que se refiere al que llama - utilizando sus propias palabras - "'choleric and quarrelsome' Doctor» habla de sus "sulky

(17) "The Pictorial Shakspere», Christian Teacher, I, n०4 (1839), pp. 322-332, "Shakespeare es un escritor cuyas bellezas poéticas no pueden ser degustadas enteramente salvo por aquellos que lo han estudiado. Como Miguel Angel -el Shakespeare de los pintores - , y como todo artista verdaderamente inspirado, nuestro poeta no despliega ninguno de aquellos encantos superficiales $y$ casi ordinarios que inmediatamente cautivan la mirada del vulgo. Sin una asidua veneración al santuario del genio, no espére nadie que se le haga partícipe de sus revelaciones. Por el contrario, será castigado por su temeridad, hallando aquellas apariencias que se manifiestan a los ojos vulgares no sólo mediocres sino repulsivas»].

(18) Blanco reconoce citar fragmentos «[...] of those which would offend those critics of the old French school, who granted but a scanty measure of Poetry to the style of the Drama; but that icy school exists only in a faint remembrance, and the lovers of poetical sublimity may now enjoy it in peace wherever they find it». ( $T$ The Pictorial Shakspere. Second notice», Christian Teacher, I, n 5 (1839), pp. 469-481, pp. 474s. [Fragmentos «de los que molestarían a aquellos criticos de la vieja escuela francesa que sólo concedian una exigua medida de poesía al estilo dramático; pero esta gélida escuela sólo existe como un débil recuerdo, y los amantes de lo sublime poético pueden ahora gozarlo tranquilamente dondequiera que lo encuentren»]. 
eyes of [...] technical critic» (19). En otro momento dice, jovial: «I am glad Dr. Johnson is already in Hades; else I should be in danger of having my head broken» (20). Luego, en un tono mucho más serio, afirma:

One could hardly expect to find these words [se refiere Blanco a una frase de Johnson: "The pretend madness of Hamlet causes much mirth»] in an English edition of Shakspere; but they are in all editions as part of the oracular judgement of a supposed giant of literature! What a total want of feeling and taste is betrayed in that single sentence!» (21)

Al margen de las diferenciaciones que el mismo Blanco White propone como encuadre inicial de su crítica, ésta puede comprenderse con arreglo a tres fundamentos temáticos de amplia tradición en sus ideas literarias. En primer lugar, en los comentarios shakespeareanos quedan definitivamente cohesionados los elementos del dilema razón-imaginación. Por otra parte, la bipolaridad naturaleza-arte se muestra en el pleno sentido que ha ido adquiriendo a lo largo de la evolución estética de Blanco. En último término, y como consecuencia de lo anterior, se desarrolla un concepto de simbolización que concluye en la afirmación del exclusivo ser ideal de la obra de arte.

El punto de partida de nuestro autor es la consideración del proceso creador en el teatro de Shakespeare. Desde el principio parece estar proponiendo Blanco una especie de mágico genio en el dramaturgo inglés, que confiere a sus obras una apariencia vívida, lo cual pudiera dar a entender que han sido elaboradas descuidadamente sobre ciertos tópicos de la escena contemporánea. Pero esto, dice el comentarista, no es en absoluto así. Los dramas shakespeareanos exigen una concepción exacta del desenvolvimiento de los caracteres antes de que el autor se enfrente a la escritura; sucede que tal concepción es de esencia simbólica, no exige una fría y precisa regulación intelectualista; de ahí la

(19) Ibid., p. 472. [«Colérico y pendenciero Doctor»] [«hoscos puntos de vista de crítico técnico"s].

(20) "The Pictorial Shakspere. Notes on Hamlet", Christian Teacher, I, $\mathrm{n}^{\circ} 6$ (1839), pp. $573-580$, p. 576. [ $« M e$ alegro de que el Dr. Johnson haya pasado a mejor vida; de otro modo estaría yo en peligro de tener quebrada la cabezan].

(21) Ibid., pp. 579s. I «Uno difícilmente podría esperar hallar estas palabras («La pretendida locura de Hamlet causa demasiada hilaridad») en una edición inglesa de Shakespeare; pero están en todas las ediciones como parte de los dogmáticos juicios de un pretendido coloso de la literatura. ¡ Qué profunda carencia de sensibilidad y de gusto se releva en esta simple frasel»]. 
marca de frescura que borra cualquier otra huella del intenso esfuerzo de concepción que ha ocupado al poeta. Así lo expone el propio Blanco:

It is true that Shakspere's historical dramas seem to have grown without effort out of the events recorded in the chronicles, or from some traditional characters established among the admirers of the theatres in earlier days than those of Shakspere. But to say that he did most carefully elaborate these compositions is a gross error, grounded on ignorance combined with a very careless reading of the historical plays. Every one of them is a fresh instance of the astonishing creative power of our bard (22). An inferior writer would be seen constantly in feverish agitation in search of incidents to complicate the plots. Shakspere, on the contrary, allows the stream either of history or tradition to waft him on, at full ease; for he is conscious of a magic spell within, by means of which creations of the highest beauty and interest shall crowd to decorate the plain line of his course. (23)

\section{En otro lugar se dirige Blanco a cierto tipo de lectores de Shakespeare}

\section{[...] for whom a judicious criticism may clear the way to a full} enjoyment of this poetical jewel: I mean those who miss the perception of its high beauties from a preconceived notion, that the Poet himself did not know what he was about to write: that the fertility of his imagination, which found an ever ready help in the flexibility and richness of his language, tempted him to pour out at random, a multitude of pictures and sentiments, drawn indiscriminately from the highest regions of poetry and the lowest of wit

(22) El sentido de most carefully elaborate hay que entenderlo a la luz de su oposición conceptual con fresh. El subrayado es mío.

(23) "The Pictorial Shakespere. Second notice», pp. 469s. ["Es verdad que los dramas históricos de Shakespeare parecen haberse construido sin esfuerzo a partir de los sucesos que se recuerdan en las crónicas o de algunos caracteres tradicionales perfectamente conocidos entre los aficionados al teatro en época anterior a la suya. Pero decir que elaboró muy cuidadosamente estas composiciones es un gran error, basado a un tiempo en la ignorancia y muy escasa lectura de las obras históricas. Cada una de ellas es un fresco ejemplo del asombroso poder de creación de nuestro bardo. Un escritor inferior habría permanecido constantemente en una febril agitación a la búsqueda de incidentes para completar la trama. Shakespeare, por el contrario, permite que las corrientes de la historia y de la tradición lo lleven con toda facilidad, porque él es consciente de un mágico hechizo por medio del cual las creaciones de la más alta belleza e interés se apresurarán a embellecer su diáfano devenir'm]. 
and satire, all which he bound together without any other plan than the distribution suggested by the five Acts of a Drama. To any one placed $[\ldots]$ on such a wrong standing point, the poem we examine [A Midsummer Night's Dream] must present the appearance of a wilderness, however beautifully decked with flowers $[\ldots]$. (24)

Por último, comentando la escena quinta del primer acto de Hamlet, apunta:

To me, this passage is an unquestionable Proof that Shakspere did not bring out the Character of Hamlet gradually; but that he conceived it perfectly and distinctly from the Beginning. And it is indeed one of the boldest Creations of Genius, as forming the Ground of a very high tragical Effect. (25)

En suma, Blanco está proponiendo la superioridad directriz del genio consciente (26). Las maravillosas apariencias de sus obras, los grandes símbolos que crea el poeta inglés exigen una profunda autopenetración intelectual. Los frutos de la capacidad imaginativa no han de surgir de un aéreo rapto mental, de una especulación en el vacio; la verdadera imaginación ha de ser tal que integre el más acentuado poder de capta-

(24) "The Pictorial Shakspere. A Midsummer Night's Dream», Christian Teacher, II, $\mathrm{n}^{\circ}$ 7 (1840), pp. $42-53$ (traducción parcial de Juan Goytisolo en Obra inglesa, pp. 319 y $326 s$. , p. 46 . [ «... a los que una crítica ajustada puede facilitar el acceso a un pleno disfrute de sus galas poéticas: me refiero a aquellos que yerran en la percepción de sus grandes bellezas a causa de la preconcebida idea de que el poeta mismo no sabia sobre qué iba a escribir; que la fertilidad de su imaginación, que encontró un recurso siempre dispuesto en la flexibilidad y riqueza de su lengua, lo impulsó a desperdigar inopinadamente una gran cantidad de escenas y sentimientos extraídos de forma indiscriminada de los más elevados ámbitos de la poesía y de los inferiores de la sátira y la ocurrencia chistosa, todo lo cual acumuló sin otro plan que la distribución sugerida por los cinco actos del drama. Para cualquiera que se halle emplazado sobre tan erroneo planteamiento el poema que examinamos / Sueño de una noche de verano] debe presentar el aspecto de una salvaje inmensidad, bien que bellamente cubierta de flores...»].

(25) "The Pictorial Shakspere. Notes on Hamlet», pp. 576s. [ «Para mi, este fragmento es una prueba incuestionable de que Shakespeare no desarrolló el carácter de Hamlet gradualmente, sino que lo concibió perfecta y distintamente desde el principio. Y ésta es, ciertamente, una de las más audaces creaciones del genio, que constituye el fundamento de un gran efecto trágicon) I.

(26) Las ideas de Blanco acerca del consciente manejo que hace Shakespeare de todos los elementos que despliega, sin ceder en ningún momento a la mera ensoñación intuitiva, tienen un paralelo en la exposiciones de Coleridge, para quien el dramaturgo, «nunca escribió nada sin designion (Apud., M. H. Abrams, El espejo y la lámpara. Teoría romántica y tradición crítica, Barcelona, 1975, p. 308). 
ción racional. Pero aún la cohesión perfecta del inicial dilema entre la razón e imaginación precisa para producirse la aparición de un nuevo dato, el de la relación entre naturaleza y arte. Partimos de una frase que parece no ser otra cosa que un arrebato de admiración: «[...] the Poet has called to life by the absolute power of his genius.) (27) Pero inmediatamente nos encontramos con lo siguiente:

True Genius brings the mind it inspires into union with the mysterious Spirit, the Soul which makes Nature, not a magazine of dead tools, but a living Unit. It is not an empty figure of speech to say that Shakspere's creations bear a strict similarity to those of Nature herself: in point of consistency, in regard to that wonderful growth which by a regular, uninterrumpted process developes the smallest seed into beings of stupendous grandeur, a strict conformity will be found between Nature and her favourite. (28)

A pesar del lenguaje que aquí utiliza Blanco no puede pasar desapercibida la explícita formulación que hace de lo que la estética idealista alemana había aportado a la ideología romántica, a saber, su concepción orgánica de la naturaleza (29). Tal concepción se había usado metafóricamente a menudo para representar el proceso creador del artista. Su más exitosa formulación se debe, en el ámbito británico, a Coleridge, que compara el crecimiento de la obra en el interior de la mente del poeta al de una planta que asimila y transforma elementos externos otorgándoles nueva vida desde sí misma. Tal es el proceso de gestación propio de lo que él llama imaginación orgánica, oponiéndola a la mera fantasía mecánica que agrupa sin más los datos proporcionados por la experiencia (30). En el presente texto de Blanco, una acción mecanicista convertiría el recurso a la naturaleza en una operación fría y numérica, como si ésta fuera un mero "depósito de instrumentos inertes»; en un

(27) "The Pictorial Shakspere. Second notice», p. 470. [«... el poeta ha convocado la vida por el absoluto poder de su genion!.

(28) lbid. p. 471. [«El verdadero genio conduce al entendimiento que inspira a la unión con el misterioso espíritu, la esęncia que causa la naturaleza - no un depósito de instrumentos inertes sino una vivida unidad. No es una vana figura de dicción decir que las creaciones de Shakespeare guardan una estricta similaridad con las de la propia naturaleza: en punto a consistencia, en relación a ese maravilloso desarrollo que por un proceso regular e ininterrumpido convierte la más pequeña semilla en una magnificencia extraordinaria, una estricta conformidad se hallará entre la naturaleza y su favoriton].

(29) Blanco cita la edición alemana de Shakespeare con versiones de A.W. Schlegel y anotaciones de L. Tieck (1821-1823); Cf., iPictorial Shakspere. A. Midsummer Night's Dream», pp. 45s. 
poeta verdadero la «más pequeña semilla», a través de un «proceso regular e ininterrumpido", se convierte en una obra plena que parece - como la naturaleza - producirse a sí misma. Tal es el sentido cabal que adquiere el dilema naturaleza-arte en la última obra crítica del escritor sevillano. La naturaleza no es percibida en el periodo romántico como término de una oposición en cuyo extremo opuesto se encuentran las creaciones del arte; al menos no siempre y necesariamente es así (31). Afortunadamente los movimientos estéticos no funcionan de forma tan simple como pretende alguna que otra malhadada corriente de historiografía literaria. Incluso el concepto de heterocosmos aplicado a las producciones de la literatura - la construcción cerrada de la obra literaria, sin otra referencia más allá de sus propias leyes constitutivas (concepto que inaugura para la estética contemporánea, siquiera teóricamente, el romanticismo) - , que pudiera parecer implica una separación insalvable del elemento naturaleza, surge de esta visión orgánica de la creación artística mediante la cual el poeta se apodera de la forma de hacer observable en el universo sensible y hace extensivos a su propia obra los principios que extrae. Tendremos ocasión de ver cómo en los últimos escritos de Blanco White aparece como natural consecuencia tal concepto.

Conozcamos previamente cómo se articula esa «estricta conformidad" entre naturaleza y artista de la que se nos habla, en lo que se refiere a la constitución de los personajes shakespearanos:

We cannot agree with those, or rather with the form of language employed by those, who say that our poet did not paint individuals, but classes. Classes are abstractions; the living stamp

(30) Coleridge, al igual que sucesivamente Blanco, «[...] convierte en foco de su examen la refutación de la antítesis general entre la naturaleza y el arte [...]) (Abrams, op. cit., p. 218). Para Coleridge, Shakespeare «[...] trabaja en el espiritu de la naturaleza, desarrollando el germen dentro, por el poder imaginativo conforme a una idea - pues como el poder de ver es a la luz; así es la idea en la mente a la ley que está en la naturaleza». (lbid., p. 314).

(31) Cf., C.M. Bowra, La imaginación romántica, Madrid, 1972, p. 24: «Algunos poetas viven enteramente en el mundo de los sueños y apenas advierten la escena visible, pero los románticos no son de esta índole. Su fuerza proviene en realidad de la manera en que arrojan una nueva luz mágica sobre la faz corriente de la naturaleza, invitándonos a buscar una explicación del irresistible atractivo que ella ejerce sobre nosotros. Todos los poetas románticos encuentran en la naturaleza su inspiración inicial. La naturaleza no lo era todo para ellos, pero ellos no hubieran sido nada sin ella, porque sólo a través de ella encontraban esos momentos de exaltación que les hacian pasar del espectáculo a la visión, para penetrar - según creían - en los secretos del universo». 
of nature is found only in individuals. Nature spoke too crearly in Shakspere's breast that he should have attempted generalizations [...]. (32)

\section{En seguida reaparece la organicidad antes aludida:}

It has been said, had Nature a concentrated consciousness, and could she be induced to give oracular answers, you might show her a single characteristic, however slight, of a human being, and she would give you his or her full character. This happens almost literally in Shakspere's personages. A name, the slightest indication of temper and mental constitution, a mere position in reference to certain events and interests, enabled him to raise up a fully developed individual, with nature's stamp of truth upon him. He was as familiar with her moral secrets as the ablest physiologist of our age showed himself with the laws of living organization. The latter, by means of a mere fragment of a skeleton, would show the whole structure of antediluvian animals, whose genera have perished; our profound moral philosopher and sublime poet gives us the exact and perfect model, the archetypal characters of men of whom nothing is left but a few historical lineaments. (33)

No ha usado el comentarista en esta descripción el término símbolo. Sin embargo, en el último artículo de la serie (sobre Midsummer Night's

(32) "The Pictorial Shakspere. Second notice», pp. 470s. [«No podemos asentir con aquellos (o mejor con la forma de lenguaje que emplean) que dicen que nuestro poeta no trazó individuos sino clases. Las clases son abstracciones; la vívida imagen de la naturaleza se halla sólo en los individuos. La naturaleza habló demasiado claro en el corazón de Shakespeare, como para que hubiera ensayado generalizaciones... 》]).

(33) Ibid., p. 471. [ "Se ha dicho que la naturaleza posee una conciencia,concentrada y que podría ser instigada a ofrecer respuestas premonitorias; se le puede mostrar una simple caracterización, por muy insignificante que ésta sea, de una criatura humana y ella devolverá su carácter completo. Esto sucede casi literalmente en los personajes de Shakespeare. Un nombre, la más sencilla indicación de temperamento y constitución mental, una mera posición con referencia a ciertos sucesos e intereses, le posibilita construir un carácter individual completamente desarrollado, con la marca natural de la veracidad sobre él. Estaba tan familiarizado con los secretos morales de la naturaleza como el más competente fisioblogo de nuestro tiempo lo está con las leyes de organización de la vida. Este último, por medio de un simple fragmento de esqueleto, podría mostrar la estructura completa de animales antediluvianos cuya especie hubiera desaparecido; nuestro profundo filosofo moral y sublime poeta nos ofrece el modelo perfecto y exacto, los caracteres arquetípicos de unos hombres de los q je nada ha quedado salvo unas breves referencias históricas»l. 
Dream, no en vano obra que se cuenta entre las más significativamente mágicas de Shakespeare por sus entrecruzamientos de los ámbitos de la realidad y el sueñol la esencia simbólica de las creaciones del poeta inglés va a ser el motivo principal del análisis. Así va a cumplirse el decisivo paso del acoplamiento entre naturaleza y arte (en el que la obra se convertirá en símbolo total de la realidad, pues ella misma es otra realidad de funcionamiento análogo) al tiempo que se producirá la plena integración entre la capacidad imaginativa y la concepción racional (en tanto que la unificación ideal entre el mundo real y el universo poético - que es la verdadera esencia de la simbolización - exige una consciencia intelectual dominadora en el artista).

El artículo comienza con la afirmación de que la pura poesía excede en belleza a cualquier representación que se haga de ella:

The opinion is gaining ground thay many [...] of Shakspere's plays lose effect in representation. [...] the pictorial embodying of some characters, $[\ldots]$ unsettle the conceptions which the lovers of Shakspere have derived from his verbal pictures. (34)

La razón de tales impresiones hay que buscarla en la inadecuación que se produce entre las visiones de la imaginación libre del lector y las restricciones materiales que ha de sufrir ese mismo lector cuando se convierte en espectador:

The representation of such compositions as the Tempest, and the one before us [A Midsummer Night's Dream, ], may if assisted by great display, amuse as a show; but the true lover of poetry cannot by this mean be satisfied for the loss of his own conceptions of the personages. In the Tragedies and historical plays the enthusiastic admirer of their author may find it difficult to accommodate his own visions to the definite shapes he sees on the stage [...]. (35)

(34) "The Pictorial Shakspere. A Midsummer Night's Dream», p. 42. [ "La opinión de que muchas de las obras de Shakespeare pierden efecto al ser representadas está ganando terreno. [...] la corporeización plástica de algunos caracteres altera las concepciones que los amantes de Shakespeare han extraido de sus escritos»].

(35) Ibid., ibidem. ["La ejecución de obras como La tempestad y la que hoy tenemos ante nosotros [Sueño de una noche de verano], servida por un gran aparato, puede divertir como espectáculo, pero el verdadero amante de la poesía dramática [sic] no se resarcirá por estos medios de la pérdida de su propia concepción de los personajes. En las tragedias y dramas históricas, el admirador entusiasta de Shakespeare experimenta cierta dificultad en acomodar su propia visión a la forma precisa que contempla en las tablas». (Trad. en Goytisolo, op. cit., p. 319)]. 
De aquí a una visión, si se permite la expresión, circunstanciadamente indefinida y vaga de la belleza hay sólo un paso. La belleza ideal, que reside en las obras musicales (36) y en la verdadera poesía, es algo de existencia pura e intocable, un símbolo ni siquiera comparable a los de las artes plásticas, pues no precisa corporeización alguna. El idealismo romántico de Blanco ha ascendido ahora hasta sus más altas cotas.

It is indeed in respect to the ideal of BEAUTY that every one who has studied this subject must perceive the superiority of pure poetry over dramatic representation and that which is obtained through the Arts of Design. True Beauty seems to have no real outline, but rather to melt, as it were, in the indefinite, the infinite. The highest works of painting and sculpture (it would appear to me) produce their wonderful effects as symbols, not as realities. In the act of looking at them, and when our vision seems to absorb all our other powers, we are constantly turning the mind's eye within us in search of a more perfect image than that which is stamped on our retina. (37)

En la escultura en particular, la emoción surge en el observador me-

(36) La relación entre la pintura y la poesía comenzó a desplazarse en Alemania en los últimos años del siglo XVIII hacia otra comparación definidora que establecía como elementos más cercanos la poesía y la música; ambas, de naturaleza inmaterial y desvinculadas de contenidos sensibles primarios (visualización), se pretendían más adecuadas a la formalización autónoma de la belleza ideal en las obras de las artes humanas (cf., Abrams, op. cit., pp. 162-173). En una carta privada de 21 de agosto de 1840, dirigida al fundador e impulsor del unitarismo norteamericano William E. Channing, introduce Blanco White algunas notas sobre la emoción musical: «[...] siento en la música un poder que no encuentro palabras para describir. Hace la música vibrar ciertas cuerdas del alma, penetra en profundidades á que ninguna otra influencia alcanza, extiende los limites de la conciencia, me deja en fin la impresión de algo misterioso, inexplicable que á nada se parece». (Apud., E. Piñeyro, "Blanco White», Bulletin Hispanique, XII (1910), pp. 72-100 y 162-200; p. 139; el subrayado es mío). La frase subrayada expresa muy claramente que para Blanco la suprema simbolización no tiene como correlato el abandono de la previsión consciente. Se conserva inédita en la Universidad de Liverpool una conferencia On musical sounds que pronunciara Blanco en Oxford en 1826.

(37) «The Pictorial Shakspere. A Midsummer Night's Dream», p. 43. [«En lo que concierne al ideal de Belleza, todo el que ha estudiado el tema advertirá la superioridad de la poesia en si sobre la representación dramática y la obtenida mediante las artes gráficas. La verdadera Belleza parece que no tenga una silueta real, sino que se funda más bien, por decirlo así, en lo indefinido, en lo infinito. Las obras maestras de la pintura y escultura producen su maravilloso efecto como símbolos, no como realidades. En el acto de contemplarlas, y cuando nuestra visión parece absorber todas nuestras demás facultades, constantemente dirigimos la mirada dentro de nosotros, en busca de una imagen más perfecta que la estampada en la retinas. (Trad. Goytisolo, op. cit., p. 319)]. 
diante el conocimiento detenido y preciso de la expresión material de cada rasgo físico; en ella

[...] motion as modifying form is the alphabet and dictionary of the language of feeling, a language which we understand more accurately in proportion as we examine its symbolical characters. Pure beauty is a mere existence which does not allow this kind of analysis. (38).

El carácter ideal de la poesía, pues, corre el peligro de desaparecer cuando ensayamos su materialización en una representación plástica. No puede ser otra, por tanto, la conclusión de Blanco: «l had much rather keep my own airy, undefined Beauty" (39).

Es ya la obra literaria como unicidad exclusiva, como heterocosmos, la que se ha manifestado ante el comentarista. Recordemos cómo Blanco decía, en el primero de estos cuatro artículos con los que se cierra su producción, que era necesaria una uasidua veneración al santuario del genio» para gozar de sus revelaciones; $y$, ¿en qué consiste tal veneración sino en la intromisión en el ámbito interno y absoluto de la obra, considerada como prolongación orgánica desde su identidad única? La mente del escritor - su consciencia, su sensibilidad, su imaginaciónes la que construye orgánicamente a partir de los materiales que extrae de sí misma y de su contexto fenoménico. Pero el resultado se constituye en supremo símbolo de la belleza, asequible sólo desde su mismidad - desde su propia ordenación natural que no precisa materialización alguna.

El sentido de la selección de los materiales dados por la experiencia vital del escritor y por la naturaleza difiere radicalmente, por supuesto, del proceso que se proponía en la estética clasicista. En ésta se trataba de una labor tendente a la ordenación mesurada de una reproducción que no podia ir más allá de los límites que el sentido común (léase, el refe-

(38) Ibid. ibidem. [ $1 . .$. el movimiento, en tanto que modifica la forma, es el alfabeto y el diccionario del lenguaje del sentimiento, un lenguaje que entendemos más adecuadamente según vamos examinando sus caracteres simbólicos. La pura belleza es una simple existencia que no consiente este tipo de análisis»].

(39) Ibid. ibidem. [«Prefiero conservar mi propia sutil e indefinida belleza»]. La excesiva brevedad de los comentarios que hace el profesor Llorens al tema que venimos tratando no es óbice para su certeza: en el teatro shakespeareano Blanco "ya no ve al poeta de la naturaleza, sino al de la metáfora). (V. Llorens, Liberales y románticos, Madrid, 1979, p. 394.) 
rente universal y abstracto naturaleza) imponía. Por el contrario, los procesos creadores descritos desde la óptica de las teorías expresivas, sobre todo si los consideramos en su última versión idealista, tienden a la integración en uno nuevo - el de la obra - del mundo sensible y del mágico (mítico, simbólico, fantástico, imaginativo, son, según cada crítico, distintos acercamientos terminológicos a idénticos fenómenos, todos ellos a la zaga de la idea de lo sublime); a la unificación de contrarios en un original e intimamente cohesionado universo.

Tal es la descripción que propone Blanco White de los diferentes planos de la posibilidad expresiva realista e imaginativa, hasta que se produce la ideal integración entre el mundo real y el poético:

The attempt to reduce these heterogeneous materials to unity, would appear perfectly absurd. How wonderful, then, must have been the power of that mind which, verifying its own grand conception of the Poet, seized both the external world and the world of Fancy, and with an ease, which has not left the slightest mark of labour, made the sauciness of Satire, the playfulness of Fancy, and the intenseness of Sentiment, unite in the most perfect harmony! In the Midsummer Night's Dream, the mind of Shakspere [...] with a kind of omnipresence, chooses, without dizziness or confusion, every object of highest beauty and cheerful interest in the vast fields of reality, of imagination, of sentiment. (40).

\section{$Y$ concluye:}

Thus in the hands of Shakspere both the real and the poetical universe become linked by feeling. (41).

(40) Ibid., pp. 48s. [ $[\mathrm{El}$ intento de reducir a la unidad tales materiales heterogéneos podría parecer del todo irrealizable. ¡Cuán extraordinario entonces debe haber sido el poder de esa inteligencia que, haciendo cierta su propia gran concepción del poeta se ha apoderado tanto del mundo aparente como del de la fantasía y, con una facilidad tal que no ha dejado la más ligera marca de su labor, ha dispuesto la gracia de la sátira, el juego de la fantasía y la intensidad del sentimiento unidos en la más perfecta armoníal En Sueño de una noche de verano la inteligencia de Shakespeare [...], con una especie de omnipresencia, sabe escoger, sin el más mínimo atisbo de confusión, todo objeto de gran belieza y vigoroso interés en los vastos campos de la realidad, de la imaginación, del sentimienton].

(41) Ibid., p. 50. [ «Así, en manos de Shakespeare, el universo real y el poético llegan a unirse mediante el sentimiento»]. Coleridge habia dicho en su Biographia Literaria: «El poeta, definido en su perfección ideal [...] difunde un tono y espiritu de unidad, que combina y funde (por asi decirlo) lo uno con lo otro, por esa fuerza sintética y mágica a la que hemos dado en propiedad exclusiva el nombre de imaginación. Esta fuerza se revela en el equilibrio o reconciliación de cualidades opuestas o discordantes...). (Apud.. Abrams, op. cit., p. 212). 
Conclusión que es a un tiempo la de la obra toda de Blanco. Los dos textos recién citados suponen la postrera manifestación de los ejes temáticos definitivos de la producción crítica del emigrado español. Naturaleza y arte, racionalidad e imaginación, han sido los conceptos fundamentadores de su devenir estético. La inicial condición de necesariedad unificadora entre naturaleza y arte ha sufrido la variación dada por la idea de lo orgánico aplicada a aquélla y la de lo simbólico aplicada a éste. Blanco ha avanzado de tal forma en un sentido progresivamente distorsionador de la previamente reducida a normas reproducción clasicista, para llegar al mantenimiento de conceptos que conllevan una pretendida más íntima captación del proceso natural y su plasmación en un producto artístico de existencia autónoma y, como tal, más idealmente reproductor de la forma de funcionamiento de una naturaleza ahora multiforme. La simplista falsedad de la concepción antitética de los dos términos del dilema ha quedado reducida a la inexistencia mediante la fructífera e incontestable integración de ambos en una relación radicalmente nueva pero no negadora absoluta de lo anterior. De ahí la sucesión, no la drástica y apresurada ruptura.

Similar es el desarrollo obrado sobre la bipolaridad razón-imaginación. La misma aparición de la componente simbólica sugiere la ausencia de conflictividad en tanto que plantea la dirección reflexiva de un proceso de tentativa sublime - vale decir, misteriosa. La apariencia mágica de la pura y excelsa belleza - tal como la piensa el último Blanco acendradamente idealista - no es otra cosa que la unificación de los ámbitos diversos, pero integrables, del mundo de lo fenoménico y de lo imaginativo; un proceso que ha sido explícitamente propuesto como regido por el genio consciente del artista.

Si puede decirse que existen tres criterios básicos para identificar la estética romántica, a saber, la imaginación como motor de lo poético, una cosmovisión orgánica y no mecanicista, y la valoración de las formas de expresión simbólica (42), podemos convenir en que nuestro autor ha hallado tales posiciones a través de un devenir que no elude la renuncia, pero tampoco la revisión y el mantenimiento reinterpretado de elementos previos. Blanco White, en fin, ha cumplido en su obra el ciclo completo que la estética europea ha llevado a cabo en la sucesión que avanza desde el clasicismo hacia la primera contemporaneidad, el romanticismo.

(42) Cf., R. Wellek, "The Concept of Romanticism in Literary History", Comparative Literature, I (1949), pp. 1-23 y 147-172; passim. 